\title{
Ethnomedicine investigation of the medicinal plants and animals in Daur, Inner Mongolia, China
}

\section{CURRENT STATUS: UNDER REVIEW}

Journal of Ethnobiology and Ethnomedicine $\triangle B M C$

Ya-Qiong Bi

Inner Mongolia Autonomous Region Academy of Traditional Medicine

Le-Tai Yi

Inner Mongolia Autonomous Region Academy of Traditional Medicine

Lei Zhang

Inner Mongolia Medical University

Ya-Hong Sun

Hailar Bureau of Science and Technology

Minhui Li

Baotou Medical College

\ prof_liminhui@yeah.netCorresponding Author

ORCiD: https://orcid.org/0000-0002-5366-8464

\section{DOI:}

$10.21203 / \mathrm{rs} .2 .18430 / \mathrm{v} 1$

\section{SUBJECT AREAS}

Translational Medicine

\section{KEYWORDS}

Daur, Hulunuir area, Plant medicines, Animal medicines, Ethnomedicine 
Abstract

Background: The classification and application of Daur medicine is an essential part of Chinese ethnomedicinal knowledge. However, the cataloging of traditional Daur medicine is still limited. As Daur medicine is gradually being replaced by traditional Chinese and modern medicine, further research is urgently needed.

Methods: We collected ethnobotany and ethnozoology data via semi-structured interviews, focus group discussions, and extensive literature reviews. Medicinal samples and specimens were collected during field investigations from June 2015 to October 2018. Attending diseases and ailments were classified according to the International Classification of Diseases 11th (ICD-11). The expression correlation of medicine classification, medicine and disease was assessed using Cytoscape 3.6.1 software. Use values were calculated to determine the importance of tradition medicinal plants and animals.

Results: We documented 30 plant species (from 19 families) and 21 animal species (from 11 families). The majority of the species were previously collected from natural habitats but they have gradually become cultivated. The most widely utilized plants were herbs (21 species), followed by shrubs (4), trees (3), and fungus (2). The most utilized groups of animals were mammals (14 species), followed by birds (5), amphibians (1), and reptiles (1). Medicines were mostly administered orally (39), but were also externally applied (11), or via both routes (9). Informants indicated that medicines were prepared via decoction (21), grinding (11), boiling (10), extraction (8), and burning (7). Most medicines are taken/consumed as a drink (37), eaten (16), or made into pills and powders (7), but also used as an anointment/wash (6), wrap/dressing (5), in the nose, eyes, and mouth (4), or as a fumigate (2). The reported traditional Daur medicines treated 76 human diseases or ailments classified under 13 disease categories, based on the ICD-11. The most commonly used medicinal species were Cervus elaphus, Cervus nippon, Capreolus capreolus, Gallus gallus, Canis lupus familiaris, Betula platyphylla, and Artemisia integrifolia.

Conclusions: A wealth of ethnobiological and ethnozoological knowledge exists, which may lead to the development of new drugs; thus, such knowledge urgently requires documentation. The information 
documented in the present study will be useful for future studies on the ethnopharmacological and traditional knowledge of the Daur region.

\section{Full-text}

Due to technical limitations, full-text HTML conversion of this manuscript could not be completed. However, the manuscript can be downloaded and accessed as a PDF.

\section{Tables}

Due to technical limitations, tables $1,2,3$, and 4 are only available as a download in the supplemental files section.

\section{Figures}

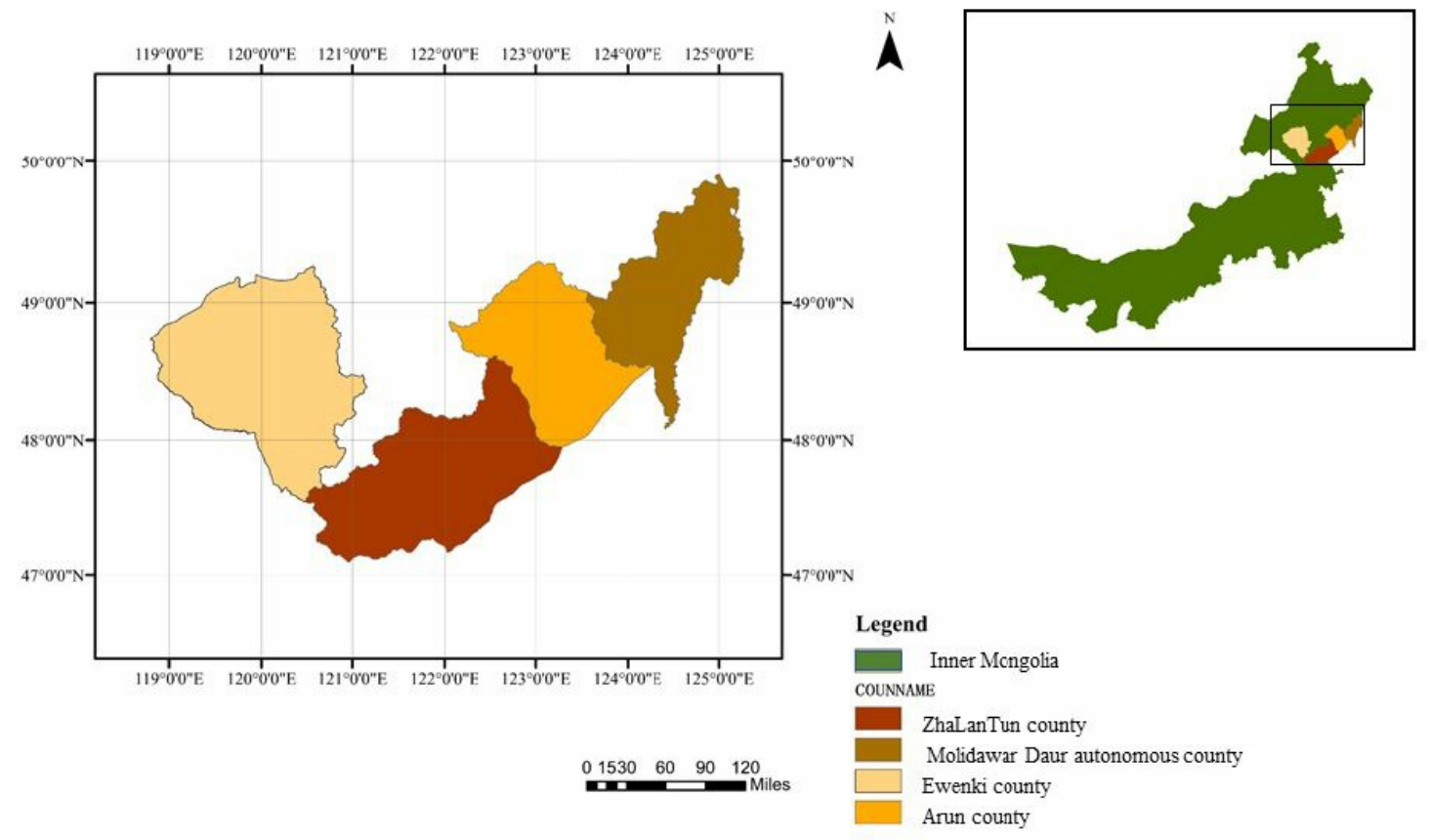

Figure 1

Map of Daur main distribution in Hulunbuir, Inner Mongolia, China. Note: The designations employed and the presentation of the material on this map do not imply the expression of any opinion whatsoever on the part of Research Square concerning the legal status of any country, territory, city or area or of its authorities, or concerning the delimitation of its frontiers or boundaries. This map has been provided by the authors. 


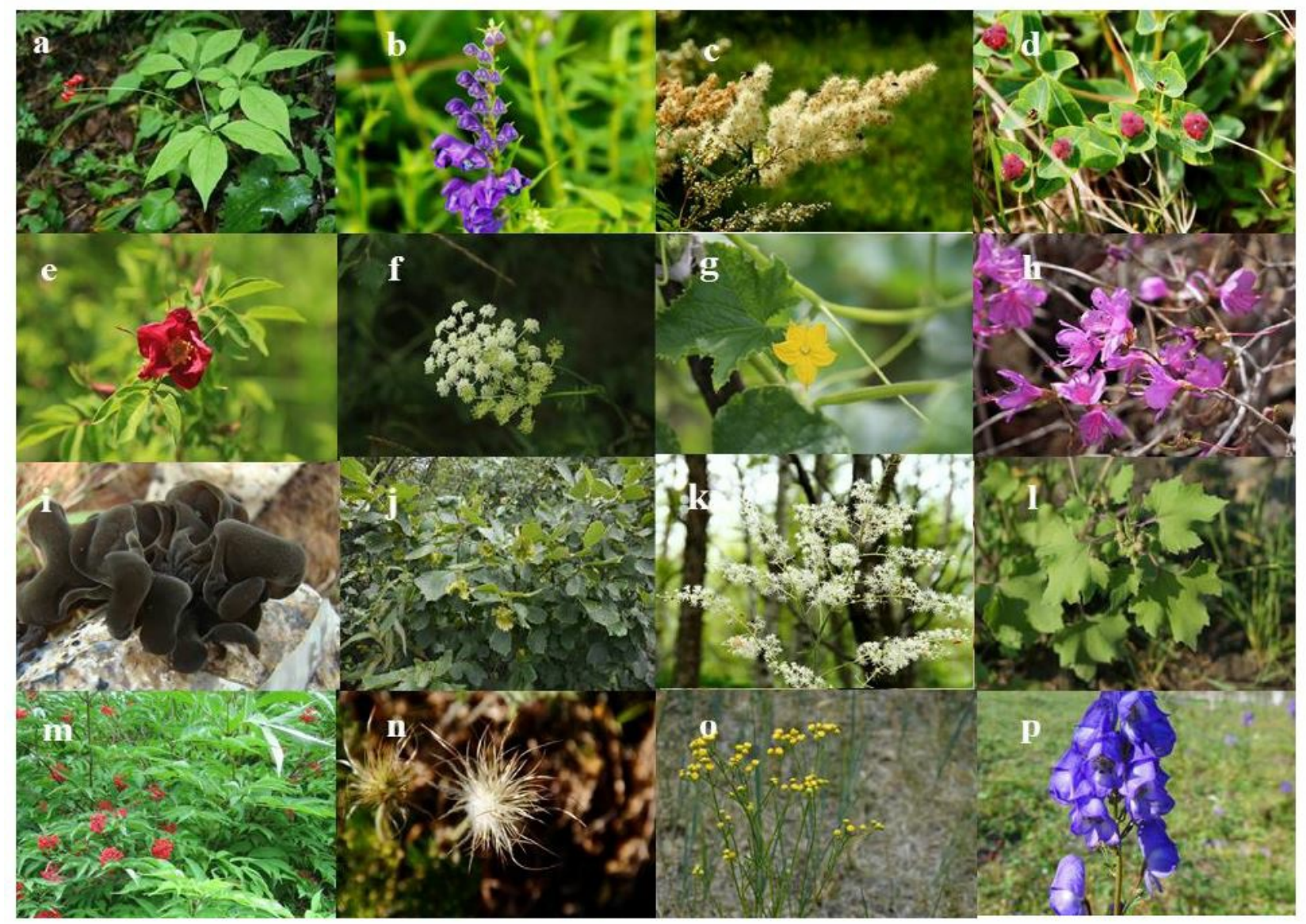

Figure 2

Parts of plants used in Daur medicine at study area $\square$ a. Panax ginseng C. A. Mey., b.

Scutellaria baicalensis Georgi, c. Sorbaria sorbifolia (L.) A. Br., d. Euphorbia fischeriana

Steud., e. Rosa davurica Pall., f. Cicuta virosa L., g. Cucumis sativus L., h. Rhododendron dauricum L., i. Auricularia auricula (L. ex Hook.) Underw, j. Corylus heterophylla Fisch., k. Cimicifuga dahurica (Turcz.) Maxim., I. Xanthium sibiricum Patrin ex Widder, m. Sambucus williamsii Hance, n. Pulsatilla chinensis (Bunge) Regel, o. Filifolium sibiricum (L.) Kitam., p. Aconitum kusnezoffii Reichb. $\square$ 
a

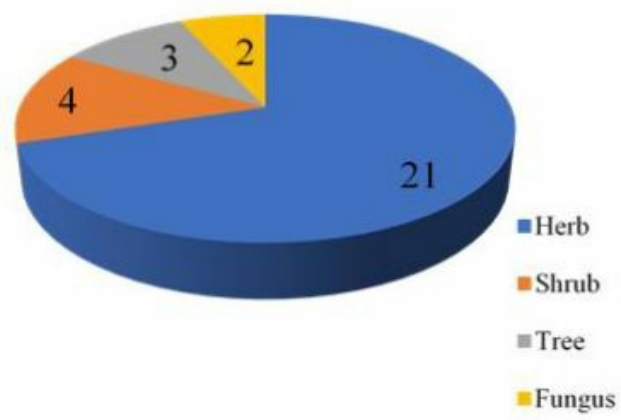

c

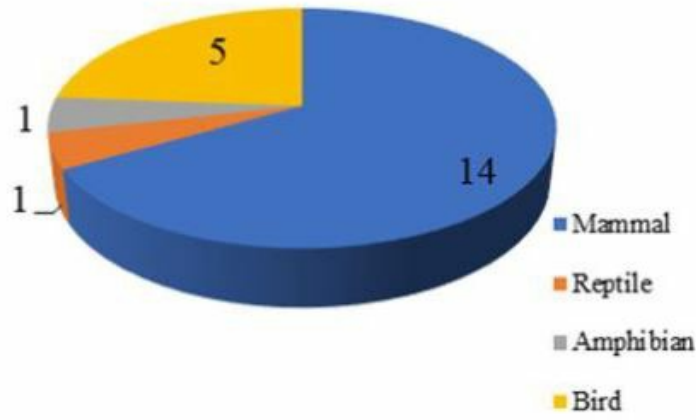

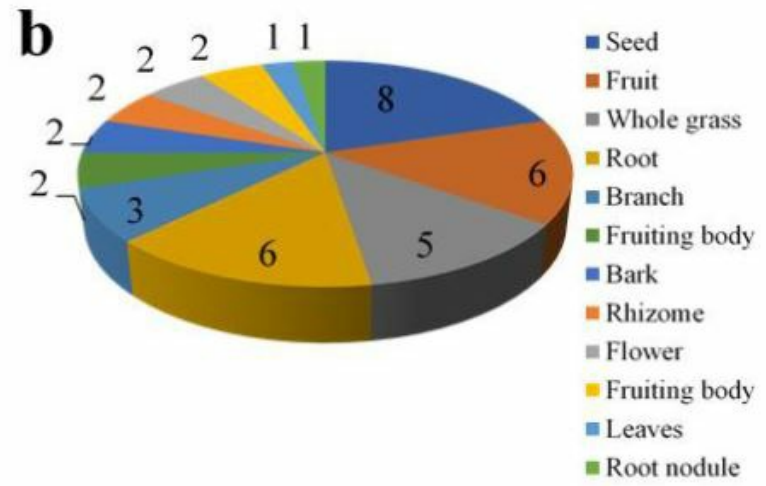

d

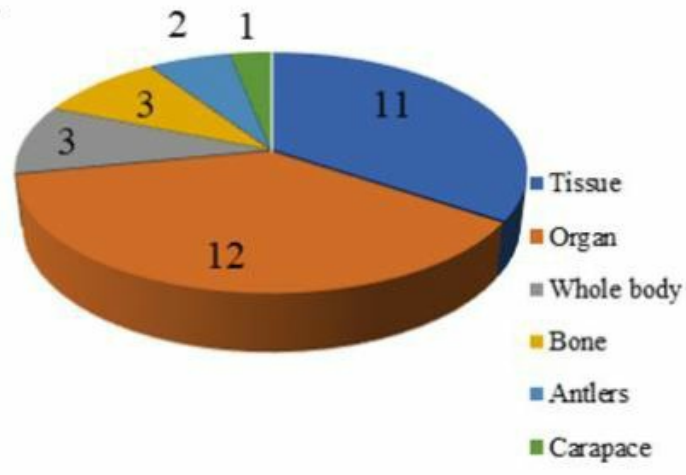

Figure 3

Graphical representation showing plant and animal species of taxonomic and various used parts (a. plant species of taxonomic; b. plants according to various used parts; c. animal species of taxonomic; d. plants according to various used parts) 


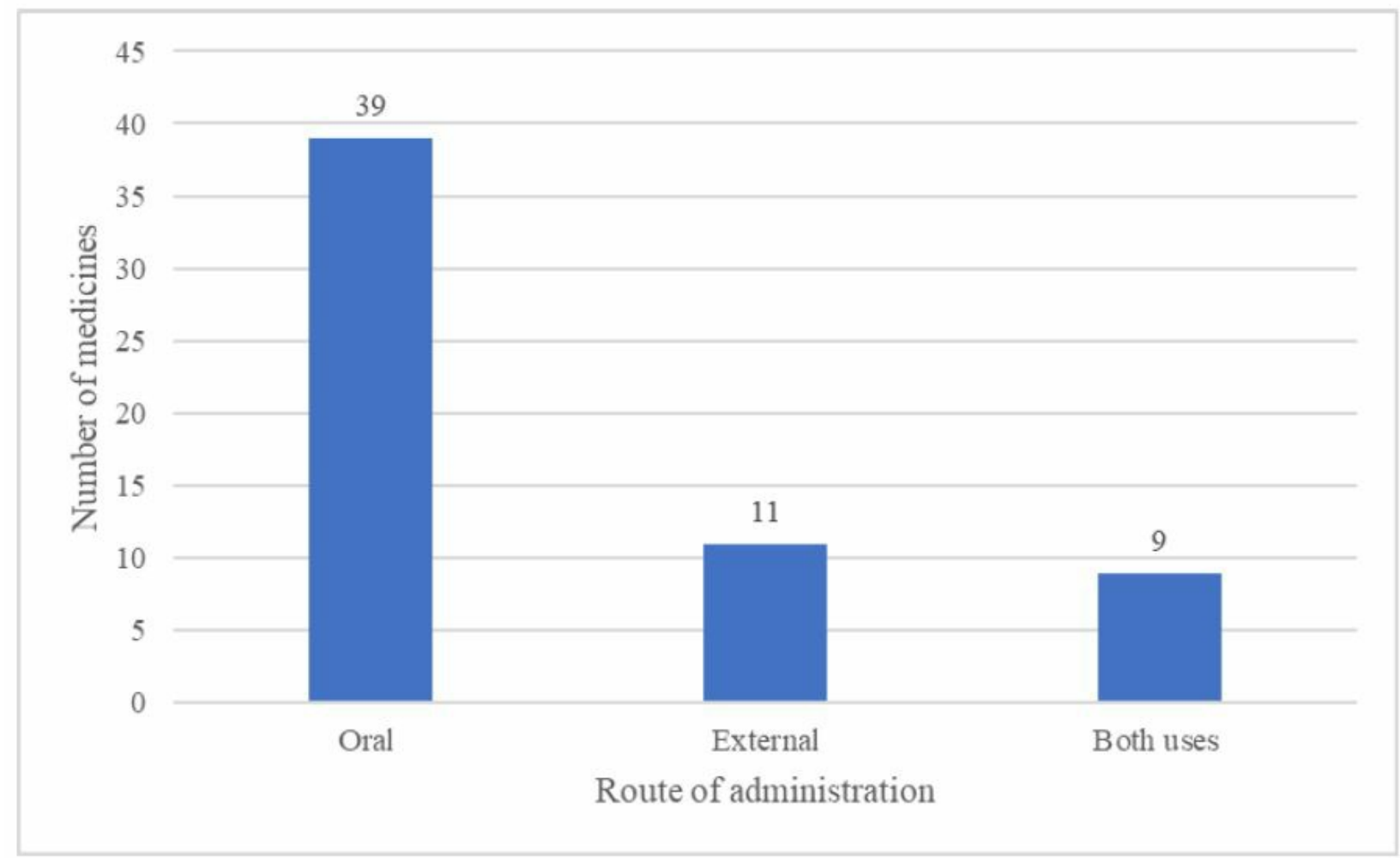

Figure 4

Rount of diversity Daur medicines 


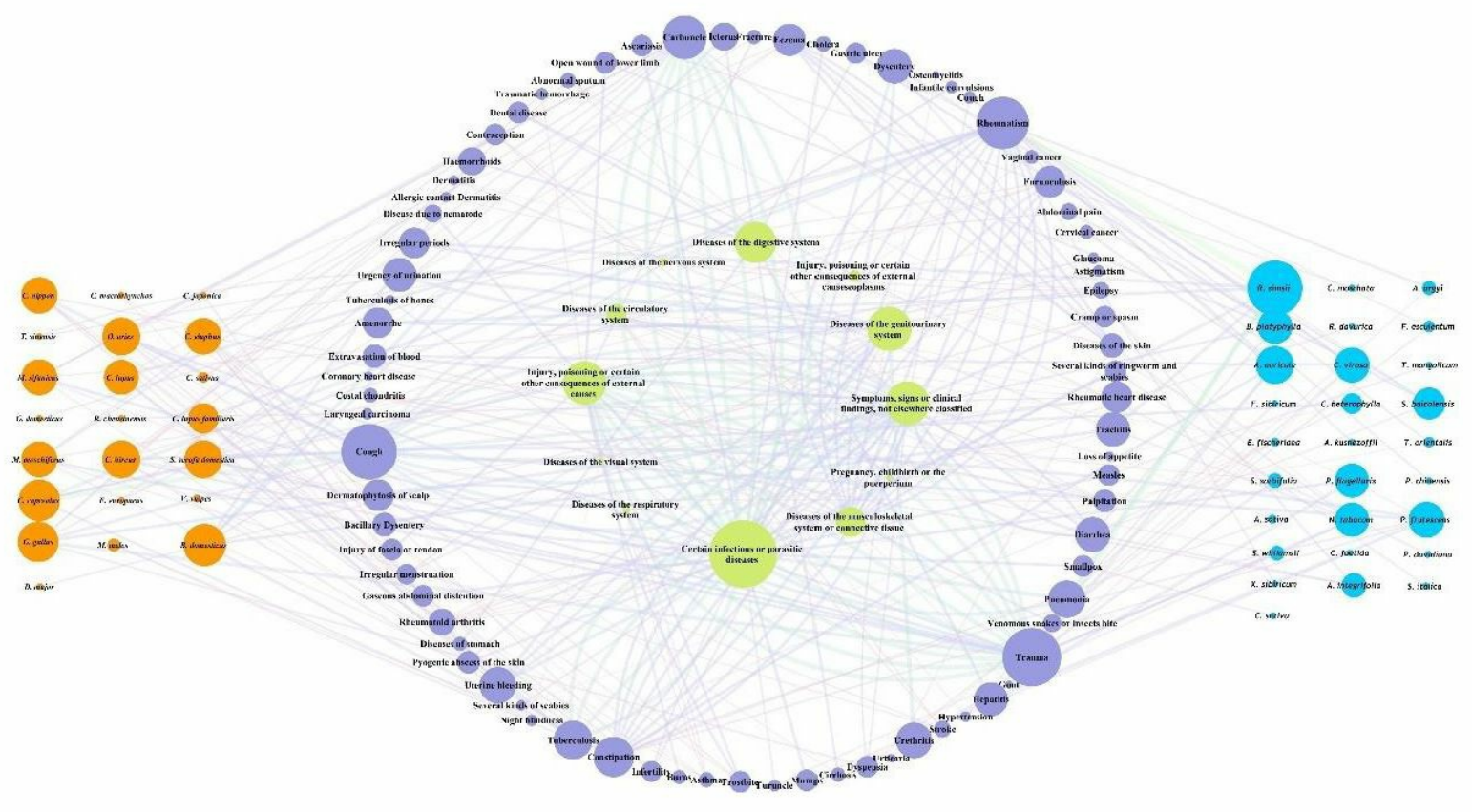

Figure 5

Network visualization of the relationship between disease and Daur medicines (The orange nodes represent plant species, the blue nodes represent animal species, the nodes represent different diseases, and the green nodes represent disease classification. The edges represent interactions. The network was built and visualized with Cytoscape.)

\section{Supplementary Files}

This is a list of supplementary files associated with this preprint. Click to download.

Tables.pdf 